\title{
An Undetected Case of Tinea Capitis in an Elderly Woman Affected by Dermatomyositis: How Trichoscopy Can Guide to the Right Diagnosis
}

\author{
Maria Vastarella Lucia Gallo Mariateresa Cantelli Paola Nappa \\ Gabriella Fabbrocini \\ Dermatology Division, Department of Clinical Medicine and Surgery, University of Naples Federico II, Naples, Italy
}

\section{Established Facts}

- Diffuse alopecia with scaly scalp dermatosis is common in patients with dermatomyositis.

- It is often treatment-resistant.

\section{Novel Insights}

- Scalp dermatomyositis requires further investigation when the patient reports no improvement with conventional therapies.

- Trichoscopy represents an essential, noninvasive technique for the evaluation and follow-up of patients with hair loss and may be an immediate, cost-effective way to guide to the right diagnosis and therapy.

- Trichoscopy may therefore be a very useful diagnostic tool in diagnosis and follow up of tinea capitis.

\section{Keywords}

Alopecia · Dermatomycosis - Tinea capitis · Trichoscopy

\begin{abstract}
Dermatomyositis (DM) is an autoimmune disease that primarily affects the skin and muscles, but may also have a systemic involvement. The frequency of scalp involvement in DM varies from 28 to $82 \%$ and is often encountered as part of a DM flare. However, it frequently presents as a treatment-
\end{abstract}

resistant disease, and patients reporting no improvement with conventional therapies require further investigation. We report a case of tinea capitis (TC) in a 79-year-old woman affected by DM with scalp involvement which did not respond to conventional therapies. Thanks to trichoscopy we could detect "comma hairs," "zigzag hairs," and "pigtail hairs" at scalp examination, made the right diagnosis of TC, and instituted the best treatment without delay.

(c) 2019 S. Karger AG, Basel
KARGER

(C) 2019 S. Karger AG, Basel

E-Mail karger@karger.com

www.karger.com/sad
Maria Vastarella

Dermatology Division, Department of Clinical Medicine and Surgery

University of Naples Federico II

Via Pansini 5, IT-80131 Napoli (Italy)

E-Mail marinavastarella@gmail.com 
Fig. 1. Before (a) and after 8 weeks of treatment (b).
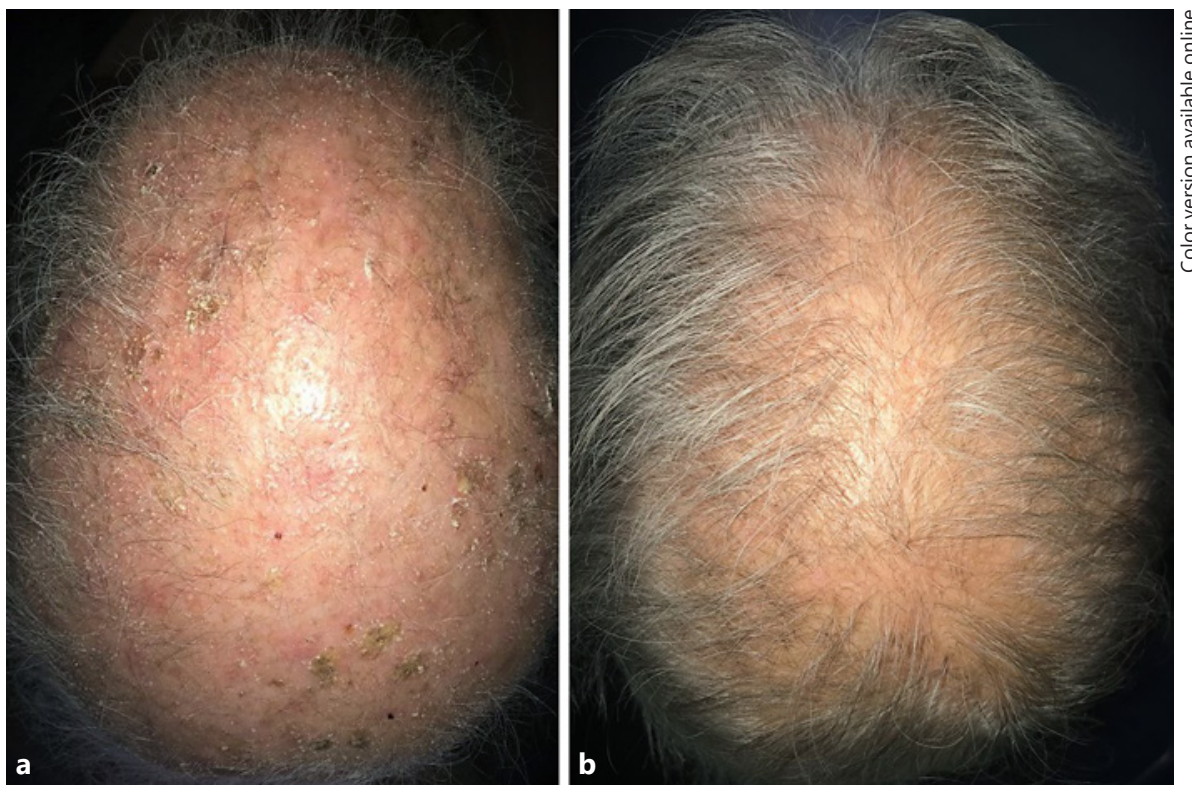

\section{Introduction}

Dermatomyositis (DM) is an autoimmune disease included in the inflammatory myopathies that primarily affects the skin and muscles, but may also have a systemic involvement [1].

Diffuse alopecia with scaly scalp dermatosis is common in patients with DM [2]. However, it often presents as a treatment-resistant disease, and patients reporting no improvement with conventional therapies require further investigation. Trichoscopy represents a valuable, noninvasive technique for the evaluation of patients with hair loss.

\section{Case Report}

A 79-year-old woman affected by DM was referred to us for the occurrence of a diffuse alopecia with scalp itching. She had a reactivation of the disease at the time of the visit and underwent therapy with methotrexate as well as oral and topical steroid that showed improved cutaneous symptoms, but no scalp improvement. Clinically the scalp presented many erythematous and scaly plaques with erythema and atrophy (Fig. 1a) typical of DM [2]. Pilotraction was positive, especially in the vertex zone. Trichoscopy using a hand-held dermoscope (DermLite DL200 Hybrid) under polarized light $(\times 10)$ showed enlarged, tortuous, and arborized vessels as well as peripilar casts, and in addition revealed a considerable number of "comma hairs," "zigzag hairs," and "pigtail hairs," with fine perifollicular scaling (Fig. 2) that suggested simultaneous tinea capitis (TC) [3, 4]. An accurate anamnesis revealed that the woman lived in the country in close contact with various farm animals, so in the presence of defined trichoscopic

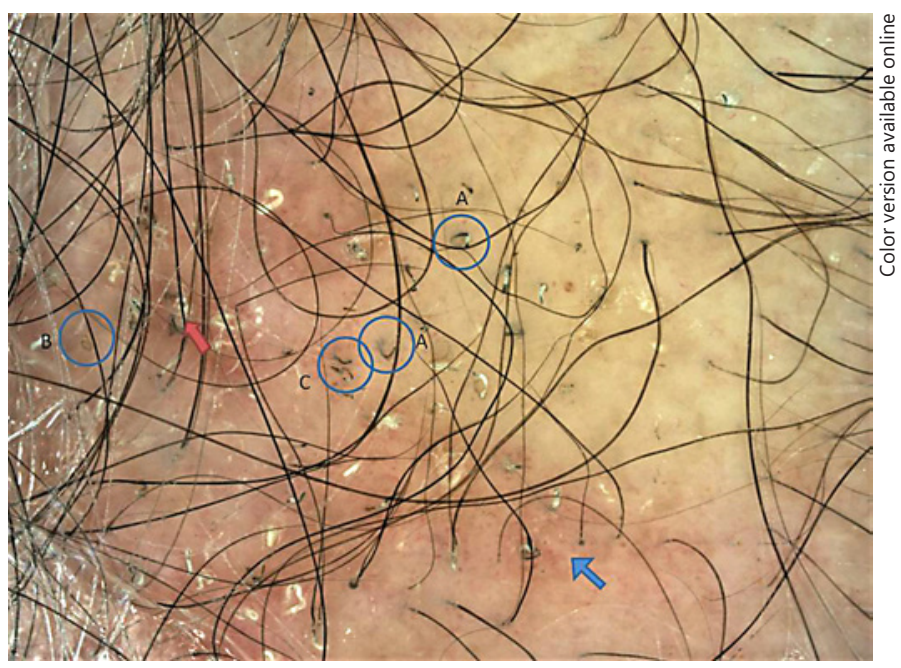

Fig. 2. Trichoscopy illustration with polarized light contact dermoscopy (DermLite DL200 Hybrid): comma hairs (A), pigtail hairs (B), and zigzag hairs (C) can be appreciated. The blue arrow highlights enlarged capillaries, while the red arrow shows peripilar casts.

features of TC we decided to perform a potassium hydroxide preparation that demonstrated abundant hyphae and spores invading the hair shafts and confirmed the diagnosis. Moreover, findings from a fungal culture of scalp scrapings identified Microsporum canis. The patient was treated with oral griseofulvin $1 \mathrm{~g}$ for 12 weeks and a topical fuchsine solution. After 8 weeks of treatment, itch was reduced and alopecia was improved (Fig. 1b), pilotraction was negative, and trichoscopy revealed complete absence of comma hairs as reported in the literature [5]. 


\section{Discussion}

The frequency of scalp involvement in DM varies from 28 to $82 \%$ and is often encountered as part of a DM flare [2]. Clinically it is characterized by atrophic, erythematous, and scaly plaques, frequently accompanied by diffuse nonscarring alopecia [2]. Major trichoscopic features in scalp DM are enlarged capillaries and peripilar casts, both present in our patient [6]. In addition, trichoscopic evaluation showed characteristic figures of "comma hairs," "zigzag hairs," and "pigtail hairs," which suggested concomitant TC $[3,4]$. Comma hairs are related to the structural weakness of the infected hairs. M. canis fungus perforates the cuticle of the hairs and causes conidia on the surface of the hairs, which bend over their lighter part [7]. Moreover, comma hairs have been hypothesized to represent cracking and bending of hyphae-filled hair shafts [7]. Normalization of trichoscopy after 8 weeks is a good prognostic sign for recovery in TC [5], and it was confirmed by mycological examination. TC is unusual in adulthood $[8,9]$, but a growing number of authors have reported an increase in TC among adults, particularly menopausal and elderly women [9]. The possible explanation of TC among adults can be attributed to hormonal changes and hair styling [8]. As the literature reports, diagnosis is delayed in most of the cases [9]. In our case the diagnosis was covered by scalp DM and by treatment with methotrexate as well as oral and topical steroids that exacerbated fungal proliferation. Although DM scalp is often resistant to treatment, in our case TC worsened the patient's symptoms.

\section{Conclusions}

Even if mycological examination is essential to confirm the diagnosis, we propose the use of trichoscopy as an immediate method to help decision-making. It represents a valuable, noninvasive technique for the evaluation and follow-up of patients with hair loss [10]. In our case it was fundamental because the clinical manifestation was not suggestive of TC and only the presence of the typical trichoscopic pattern of TC encouraged us to carry out a mycological examination.

\section{Statement of Ethics}

The authors state that the subject gave informed consent for publication of photos and details of her case.

\section{Disclosure Statement}

The authors have no conflicts of interest to declare.

\section{References}

1 Bogdanov I, Kazandjieva J, Darlenski R, Tsankov N. Dermatomyositis: current concepts. Clin Dermatol. 2018 Jul-Aug;36(4): $450-8$.

2 Tilstra JS, Prevost N, Khera P, English JC 3rd. Scalp dermatomyositis revisited. Arch Dermatol. 2009 Sep;145(9):1062-3.

3 Slowinska M, Rudnicka L, Schwartz RA, Kowalska-Oledzka E, Rakowska A, Sicinska J, et al. Comma hairs: a dermatoscopic marker for tinea capitis: a rapid diagnostic method. J Am Acad Dermatol. 2008 Nov;59(5 Suppl): S77-9.
4 Elghblawi E. Idiosyncratic Findings in Trichoscopy of Tinea Capitis: Comma, Zigzag Hairs, Corkscrew, and Morse Code-like Hair. Int J Trichology. 2016 Oct-Dec;8(4):180-3.

5 Vazquez-Lopez F, Palacios-Garcia L, Argenziano G. Dermoscopic corkscrew hairs dissolve after successful therapy of Trichophyton violaceum tinea capitis: a case report. Australas J Dermatol. 2012 May;53(2):118-9.

6 Jasso-Olivares JC, Tosti A, Miteva M, Domínguez-Cherit J, Díaz-González JM. Clinical and Dermoscopic Features of the Scalp in 31 Patients with Dermatomyositis. Skin Appendage Disord. 2017 Aug;3(3):119-24.

7 Lin Y, Li Y. The dermoscopic comma, zigzag, and bar code-like hairs: markers of fungal infection of the hair follicles. Zhonghua Pifuke Yixue Zazhi. 2014;32(3):160-3.
8 Calabrò G, Patalano A, Fiammenghi E, Chianese C. Tinea capitis in Campania, Italy: a 9-year retrospective study. G Ital Dermatol Venereol. 2015 Aug;150(4):363-7.

9 Morell L, Fuente MJ, Boada A, Carrascosa JM, Ferrándiz C. [Tinea capitis in elderly women: a report of 4 cases]. Actas Dermosifiliogr. 2012 Mar; 103(2):144-8.

10 Lacarrubba F, Verzì AE, Micali G. Newly described features resulting from high-magnification dermoscopy of tinea capitis. JAMA Dermatol. 2015 Mar;151(3):308-10. 